\title{
Selective laser sintering and its application in biomedical engineering
}

\begin{abstract}
Bin Duan and Min Wang
Rapid prototyping (RP) technologies, which are based on computer-aided design and computer-aided manufacturing, are widely employed in traditional industries. They are capable of achieving extensive and detailed control over the architecture of objects to be formed and therefore are increasingly used in the biomedical engineering field. Selective laser sintering (SLS), a versatile RP technique, uses a laser beam to selectively sinter powdered materials to form three-dimensional objects according to designs that can be based on data obtained from computer-based medical imaging technologies. In this article relating to biomedical applications, the principle, materials, machine modification, and parameter optimization for SLS are reviewed. Biomedical applications of SLS, especially in the fabrication of tissue engineering scaffolds and drug/biomolecule delivery vehicles, are presented and discussed. SLS exhibits great potential for many applications in biomedical engineering.
\end{abstract}

\section{Introduction}

As a designed manufacturing technology, solid free-form fabrication (SFF) or rapid prototyping (RP) has drastically improved our ability to fabricate objects with customized designs, complex geometries, precise dimensions, and controllable architecture. In fact, RP is a group of techniques that can generate a physical model directly from computer-aided design (CAD) data in a layer-by-layer manner, where each layer is the shape of the cross-section of the model at a specific level. ${ }^{1}$ In applying RP techniques in biomedical engineering, the data used to design and fabricate objects are usually based on data from CADs, computer-based medical imaging technologies such as magnetic resonance imaging (MRI) and computer tomography (CT), or other computer-based technologies. ${ }^{2}$ Computer-based data are converted to an STL file format (which is a standard format for RP techniques), STL being derived from "stereolithography," the oldest RP technology. The computer-generated two-dimensional layers are then assembled, and a concrete model is created, starting from the bottom and proceeding upward. In production, each layer is bonded to the previous layer, producing a solid object based on the design.

Based on their working principles, RP techniques can be divided into several categories: laser polymerization-based techniques, such as stereolithography apparatus and two-photon polymerization; nozzle deposition-based techniques, such as fused deposition modeling and three-dimensional (3D) plotting; and powder-based techniques, such as 3D printing (3DP) and selective laser sintering (SLS)., ${ }^{3,4}$ Generally, each technique is suitable for a specific material (or group of materials) or a specific form of the material and has its own advantages and disadvantages.

Two decades ago, tissue engineering emerged as a promising alternative to using autografts, the gold standard in current surgery, and allografts for tissue repair and regeneration. There are several strategies in tissue engineering, including cell-based tissue engineering, factor-based tissue engineering and scaffold-based engineering. For cell-based tissue engineering, autologous or allogeneic cells are first isolated and then injected in single cell suspensions at the targeted sites of patients, or they can be manipulated by cell assembly into multicellular spheroids or cell sheets for transplantation in patients. Factor-based tissue engineering is conducted by the injection of biomolecules (normally in a carrier), such as growth and differentiation factors, or genes to targeted locations in patients. For scaffold-based tissue engineering, cells and/or biomolecules are encapsulated within the matrices or seeded onto the surface of three-dimensional (3D) scaffolds for tissue regeneration. ${ }^{4}$ The scaffolds serve as extracellular matrix to direct cell adhesion, proliferation and differentiation and thus promote new tissue formation. With the emergence and development of tissue engineering, various biomaterials and RP techniques are being investigated for fabricating porous scaffolds with controlled 
architectures (pore size, shape, interconnectivity, porosity) and properties (biological, mechanical), targeting the regeneration of different tissues. ${ }^{5-7}$

SLS employs a laser beam, usually a $\mathrm{CO}_{2}$ laser, to selectively sinter thin layers of powdered polymers or polymer-based composites, forming solid 3D objects with macro- and microscale features. SLS has several advantages in forming designed objects, including high part accuracy, material versatility, and no need for part support during manufacture because the material not fused together by the heat generated by the laser beam provides a good support for the object being sintered. It has thus been used for constructing models for surgical planning and for medical device prototyping. In addition, SLS is highly capable of producing objects with irregular shapes, including structures containing channels and overhanging features. ${ }^{1}$ Therefore, SLS is a very attractive RP technique for the fabrication of tissue engineered scaffolds with controlled pore structure, pore size, and customized scaffold architecture. In this article, the working principle of SLS and modifications of SLS machines are reviewed. Materials used in SLS and optimization of SLS parameters for fabricating good-quality objects are highlighted. Biomedical applications of SLS, especially in tissue engineering and drug/biomolecule delivery, are presented and discussed.

\section{Selective laser sintering SLS process and modifications of commercial SLS machines}

SLS was developed and patented by Deckard and Beaman in the mid-1980s. It was commercialized in 1987 by DTM Corporation, which was acquired by 3D Systems in 2011. Like most RP techniques, SLS is an additive fabrication process that allows the fabrication of objects from CAD files. The architecture (external and internal of structures) of objects can be designed using professional 3D-drawing computer software, described by mathematical equations, or derived from data from imaging techniques such as MRI and CT (Figure 1a). The CAD file with information on geometry and dimensions of an object is converted into an STL file and input into an SLS machine. The machine then uses a fine-powder material to fabricate the 3D object according to the designed model (Figure 1b-d). During SLS, the powder material is heated up by a laser beam such that the surface tension of individual particles in the powder is overcome and the selectively sintered powder fuses together, solidifying a thin layer and then building a solid 3D object layer-by-layer.

Before the powder material is selectively sintered, the entire part bed (the area that contains both the powder being sintered and the sintered part) of the machine is heated to just below the melting temperature of the material (or near the glass transition temperature of amorphous polymers) in order to minimize thermal distortion and facilitate fusion of the sintered layer. The laser beam then scans over the cross-section on the powder surface according to the cross-sectional profiles from the slice data, heating up the powder and causing particles to fuse together to form a solid layer. After one layer is finished, the part bed is lowered, and one of the powder tanks, which is filled with the powdered raw material, is raised. A new layer of powder is then spread by a roller, and the selective sintering process is repeated. The powder that is not scanned by the laser beam remains in place to serve as the support to the next layer of powder, then is removed and recycled once fabrication of the object is completed.

In a commercial SLS machine, an SLS process typically requires a powder supply of at least one-third of the powder tank volume. Therefore, an unmodified commercial SLS machine is not material-efficient for producing objects with small dimensions and small features or for building tissue engineering scaffolds using expensive materials. In our research, in order to reduce the consumption of biopolymer or biocomposite powders for constructing tissue engineering scaffolds via SLS, modifications were made to a commercial SLS machine (Sinterstation 2000). ${ }^{8}$ A miniature sintering platform was designed, fabricated, and installed in the build cylinder of Sinterstation 2000 (Figure 2c). The miniature platform consisted of a miniature sintering platform (Figure 2d) and two powder tanks similar to the commercial machine but with much smaller dimensions (the size of powder tanks in a commercial Sinterstation 2000 SLS system is $325 \times 250 \times 370 \mathrm{~mm}^{3}$, and the size of miniature power tanks is $60 \times 60 \times 75 \mathrm{~mm}^{3}$ ). After the modifications, only small amounts of powder were needed to fill the miniature powder tank. Similarly, a compact adaptation device for a Sinterstation 2500 machine was developed, which permitted the transfer of the motion of the part bed of the SLS machine onto the part bed of the compact device. ${ }^{9}$

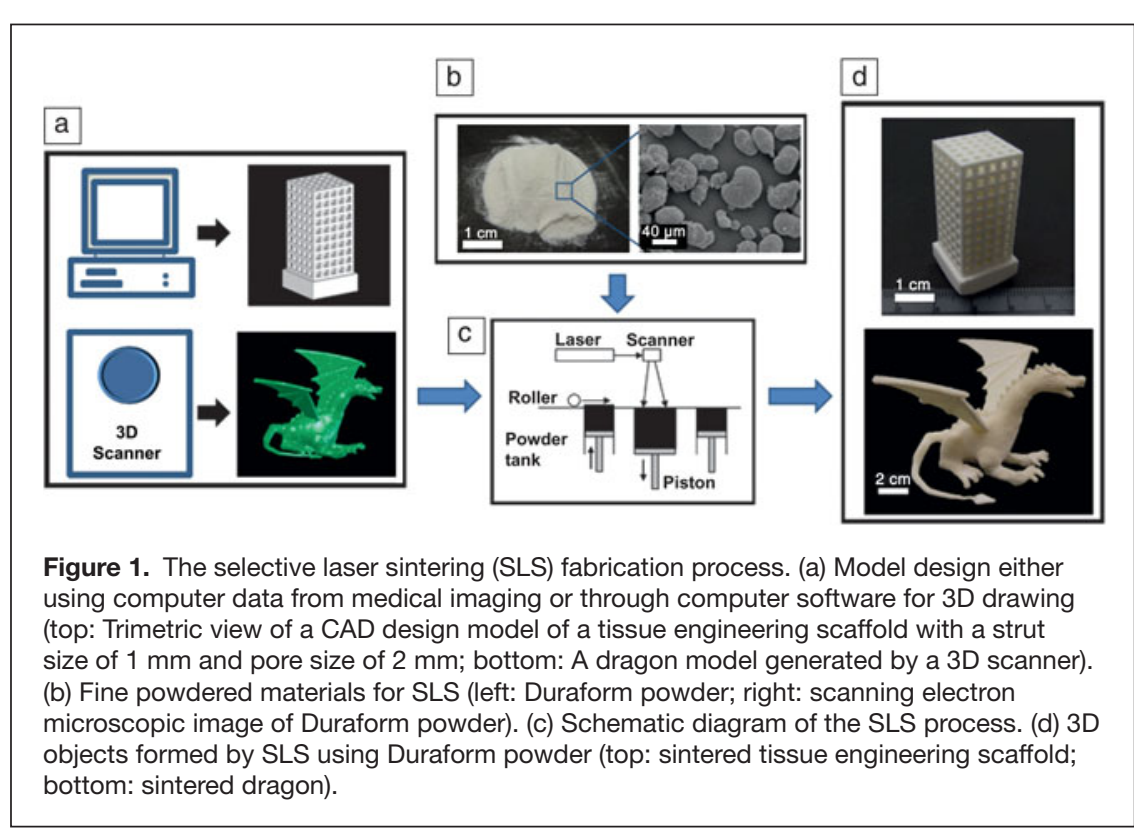




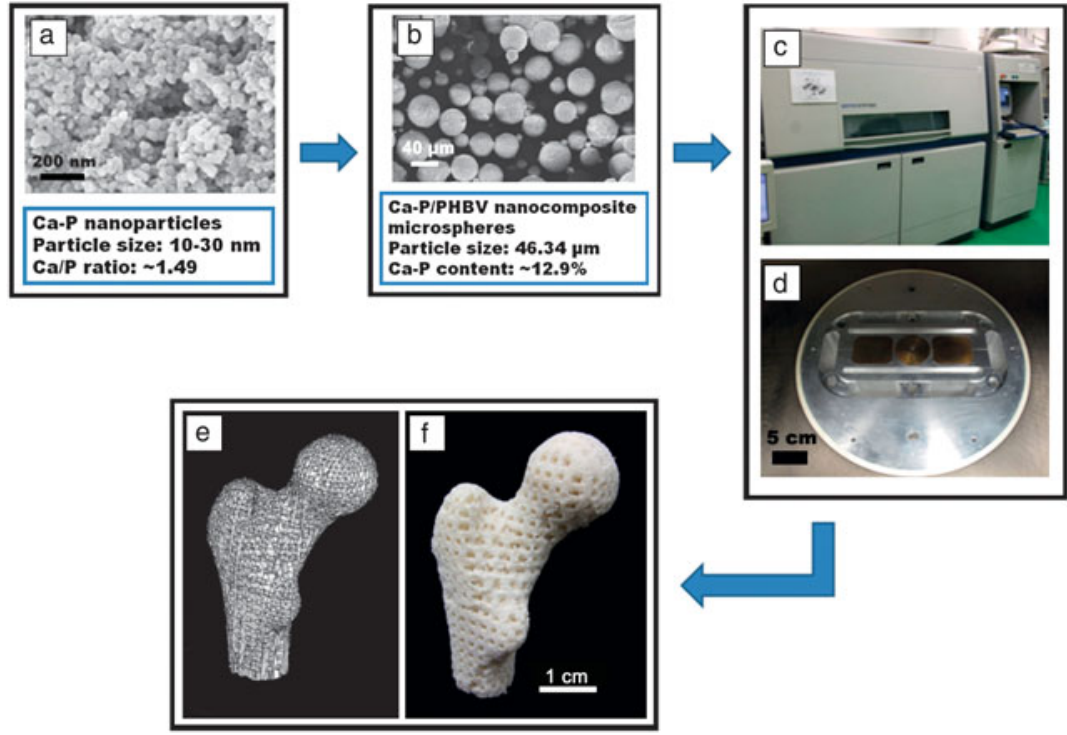

Figure 2. Selective laser sintering (SLS) fabrication of Ca-P/PHBV nanocomposite scaffolds. (a) Scanning electron microscopic (SEM) image of Ca-P nanoparticles. (b) SEM image of Ca-P/PHBV nanocomposite microspheres; (c) Sinterstation 2000 SLS machine. (d) Miniature sintering platform installed in the modified Sinterstation 2000 machine. (e) A porous scaffold model based on the 3D model of the proximal end of a human femur reconstructed from computed tomography images. (f) Sintered Ca-P/PHBV nanocomposite scaffold. (Real scaffold size: two-fifths of the design model.)

Apart from conventional SLS in which polymeric powders absorb infrared laser radiation $(\lambda=10.6 \mu \mathrm{m})$, resulting in volumetric absorption by the whole polymeric particle, near-infrared laser radiation $(\lambda=0.97 \mu \mathrm{m})$ can also be used to melt the surface of polymer particles. ${ }^{10}$ Surface selective laser sintering (SSLS) thus makes it possible to melt the surface layer rather than the whole polymer microparticle and sinter the particles into $3 \mathrm{D}$ objects. SSLS is suitable for sintering thermally unstable polymers (e.g., poly(lactic-co-glycolic acid) [PLGA]) and for fabricating scaffolds with delicate biomolecules incorporated in them. ${ }^{11}$

\section{Materials for SLS in biomedical engineering}

Many materials, from polymers to metals to ceramics, can be processed by SLS for general engineering or biomedical applications. In theory, any material that can be processed into powder and can be sintered with heat may be used in SLS. In practice, seven types of materials, including wax, polycarbonate, nylon (standard nylon, fine nylon, fine nylon medical grade, and nylon composite) and acrylic, which are all commercially available, are often used. ${ }^{12}$ Normally, $\mathrm{CO}_{2}$ lasers with a wavelength of $10.6 \mu \mathrm{m}$ are used for sintering polymers or materials with low melting temperatures. Nd:YAG lasers with a wavelength of $1.06 \mu \mathrm{m}$ can outperform $\mathrm{CO}_{2}$ lasers for metallic and ceramic materials, which absorb much better at short wavelengths. ${ }^{13}$ Pure Ti, Ti-6Al-4V, and NiTi alloy (Nitinol) were successfully sintered into 3D scaffolds using Nd:YAG lasers. ${ }^{14,15}$ Bioceramics such as hydroxyapatite (HA) could also be sintered to form customized implants for bone tissue repair. ${ }^{16,17}$ The type of laser can affect the properties (mechanical properties, density, surface texture) of sintered objects.

Powdered materials for SLS should have appropriate particle sizes and have good flowability for spreading on the part bed of the SLS machine. Materials with particle sizes of $10-150 \mu \mathrm{m}$ and with a spherical shape are preferred. ${ }^{18}$ The polymer particle size was found to have significant effects on scaffold properties..$^{19}$ Commercial SLS powder materials can be used directly or mixed with materials such as carbon black to form composite powders for SLS. For fabricating composite scaffolds for bone tissue repair, bioceramics such as HA have been dry-blended with a polymer and then made into a composite in an extruder. ${ }^{20}$ The extruded composite was subsequently pelletized and powdered for SLS. Using dryblended bioceramic-polymer mixtures directly for SLS was also attempted. ${ }^{21}$ Another strategy was to fabricate polymer microspheres or composite microspheres and use the microspheres for 3D scaffold fabrication. ${ }^{8}$ As shown in Figure 2a, calcium phosphate (Ca-P) nanoparticles with sizes in the range of $10-30 \mathrm{~nm}$ were first synthesized. Nanocomposite microspheres consisting of Ca-P nanoparticles and biodegradable poly(hydroxybutyrate-cohydroxyvalerate) (PHBV) polymer matrix, as shown in Figure 2b, were then made using the solid-in-oil-in-water $(\mathrm{S} / \mathrm{O} / \mathrm{W})$ emulsion-solvent evaporation technique. The nanocomposite microspheres were finally sintered into a scaffold in the shape of the proximal end of a human femur using a model reconstructed from CT images (Figure 2e-f).

\section{SLS parameters and their optimization}

In SLS, several parameters, including laser power, scan spacing, layer thickness, part bed temperature, scan speed, and roller speed, can significantly affect the quality of sintered objects. In order to obtain $3 \mathrm{D}$ objects with accurate dimensions as designed, good structural and handling stability, and sufficient mechanical properties, SLS parameters need to be studied and optimized. For parameter optimization, the one-factor-at-a-time method for experimentation, in which one SLS parameter is varied at a time with the other parameters being kept constant, is usually employed. ${ }^{21}$ However, results obtained through this method do not provide information about potential interactions among two or more factors, and hence do not reflect the real situation. To be closer to the actual sintering process, the factorial design methodology can be employed to evaluate the main effects and also interactions of joint factor effects on the response variables. Partee et al. used the two-level factorial design of experiments (DOE) technique to optimize SLS parameters for making poly ( $\varepsilon$-caprolactone) (PCL) tissue engineering scaffolds. ${ }^{22}$ Using the optimized parameters, solid PCL samples 
and porous PCL scaffolds with 1D, 2D, and 3D orthogonal, periodic porous architectures were produced via SLS. ${ }^{23}$ Similarly, our group considered three important aspects of scaffolds, namely structure and handling stability, dimensional accuracy, and compressive properties, and used DOE with three factors (laser power, scan spacing, and layer thickness) and three levels (high, medium, and low values of respective factor) to optimize SLS parameters for fabricating Ca-P/PHBV nanocomposite scaffolds. ${ }^{24}$ With optimized SLS parameters, 3D porous structures or scaffolds with complex interior and exterior geometries could be produced..$^{25}$

\section{Applications of SLS in biomedical engineering}

SLS can have many applications in biomedical engineering. An overview of the biomedical applications is presented, highlighting the use of SLS for tissue engineering scaffolds and drug/ biomolecule delivery systems.

\section{Tissue engineering scaffolds}

Traditional treatments for diseased or traumatized human body tissues involve the use of autograft, allograft, and biomaterials (natural or synthetic, including biocompatible polymers, metals, ceramics, and composites) for tissue repair. However, there are major limitations such as shortage of donor tissue, graft rejection, and lack of bioactivity. As a promising new strategy, tissue engineering emerged and involves the creation of biological substitutes that integrate with the host and evolve into newly formed tissue at the repair sites. ${ }^{26}$ For scaffold-based tissue engineering, controlling the macro- and micro-architecture of the scaffold and hence fulfilling a customized design with complex anatomic shape is of significant importance for the clinical application of the scaffold. ${ }^{27}$ In scaffold fabrication, in contrast with non-designed manufacturing techniques such as solvent casting/porogen leaching, freeze-drying, and gas foaming, designed manufacturing techniques such as SLS make it possible to achieve extensive and detailed control over scaffold architecture. ${ }^{28}$

Biopolymers (degradable or non-degradable), ceramics, and composites can be processed into scaffolds via SLS for bone tissue repair or bone tissue engineering. Rimell and Marquis used a simplified SLS apparatus to sinter ultrahigh molecular weight polyethylene implants. ${ }^{29}$ Other powdered non-degradable polymers such as polyetheretherketone (PEEK) have also been sintered. ${ }^{30}$ However, single-component biomaterials cannot fully fulfill the requirements for bone graft substitutes. Therefore, composite scaffolds based on nondegradable polymers and HA, including HA/PEEK and HA/ high-density polyethylene (HDPE), were made via SLS. ${ }^{31,32}$ HA/HDPE scaffolds were intended as customized maxillofacial implants. HA in the composite scaffolds enhanced cell proliferation, increased alkaline phosphatase activity, and produced more osteocalcin (a biochemical marker for new bone formation). ${ }^{33}$ In the HA/PEEK system, different amounts of HA were incorporated, and scaffolds had well-defined pore interconnectivity. ${ }^{34}$ By varying the HA amount, the porosity, mechanical properties and bioactivity of HA/PEEK scaffolds were controlled.

Within the conventional notion of tissue engineering, tissue engineering scaffolds should be biodegradable, and the degradation rate should be synchronized with the rate of neotissue formation. Therefore, for tissue engineering scaffold fabrication, the majority of research focuses on biodegradable polymers or their composites. The most widely used biodegradable polymer for scaffolds formed by SLS is probably PCL, due to its good biocompatibility and low melting temperature..$^{35}$ Williams et al. pioneered the fabrication of PCL scaffolds via SLS. ${ }^{36}$ The sintered PCL scaffolds, with an orthogonal interconnected pore design, were seeded with human gingival fibroblastic cells and subcutaneously implanted in immunocompromised mice. Micro-CT data and histological images showed that bone formed on scaffolds or inside the pores of scaffolds. Ciardelli et al. used SLS to sinter blended microparticles of PCL and a polysaccharide (starch, dextran, or gelatin) and systematically studied the morphology, thermal behavior, and cell compatibility of the composite scaffolds formed. ${ }^{37}$ Other biodegradable polymers, such as poly(vinyl alcohol) (PVA), poly(L-lactic acid) (PLLA), and PLGA, were also successfully processed into scaffolds via SLS. ${ }^{38,39}$ Bioceramics such as HA can be incorporated into biodegradable polymers to form composite bone tissue engineering scaffolds. Blends of PCL and HA were used to fabricate tissue engineering scaffolds via SLS. ${ }^{21}$ Good sintering results were achieved for these HA/PCL composite scaffolds by optimizing SLS parameters, and the scaffolds exhibited both in vitro bioactivity and in vitro cell compatibility. Other composite scaffolds such as HA/PVA and HA/PLGA have also been made via SLS. ${ }^{39,40}$

The paradigm has shifted from conventional fabrication technologies to microtechnology and now toward nanotechnology for improving the mechanical and biological properties of biomaterials. For example, nanostructured $\mathrm{Ca}-\mathrm{P}$ and nanocomposites incorporating nano-sized Ca-P provided better osseointegration as compared to their larger particle size counterparts owing to their bioresorbability and high surface area. ${ }^{41,42}$ For $\mathrm{Ca}-\mathrm{P} / \mathrm{PHBV}$ and $\mathrm{Ca}-\mathrm{P} / \mathrm{PLLA}$ nanocomposite scaffolds, in order to homogeneously incorporate $\mathrm{Ca}-\mathrm{P}$ nanoparticles, nanocomposite microspheres consisting of nano-sized $\mathrm{Ca}-\mathrm{P}$ and a PHBV or PLLA matrix were fabricated first. ${ }^{43}$ The nanocomposite microspheres then served as raw materials for SLS in the fabrication of nanocomposite scaffolds. ${ }^{43,44}$

Apart from bone tissue engineering scaffolds, SLS can also be used to fabricate scaffolds for the repair or regeneration of other tissues. Liu et al. blended epoxy resin E-12 (as a binder) with $\mathrm{K}_{2} \mathrm{O}-\mathrm{Al}_{2} \mathrm{O}_{3}-\mathrm{SiO}_{2}$ series dental glass-ceramic, forming fine powder composites, and then manufactured dental restoration devices using SLS. ${ }^{45}$ PCL scaffolds with tailored architecture and mechanical properties were fabricated via SLS to accommodate $\mathrm{C} 2 \mathrm{C} 12$ myoblast cells for cardiac tissue engineering. ${ }^{46}$ To engineer implantable liver tissues, Huang et al. designed a PCL scaffold with a 3D branching and joining flow-channel network and fabricated the scaffold using SLS. ${ }^{47}$ 


\section{Drug/biomolecule delivery vehicles}

SLS can also be used to fabricate 3D devices for drug or biomolecule incorporation and delivery. Leong et al. demonstrated the fabrication of drug delivery devices (DDDs, in the form of cylinders $9 \mathrm{~mm}$ long) by selective laser sintering nylon powders and used methylene blue dye as the model drug. ${ }^{48}$ In order to control the release profile, two features, namely porous microstructure and dense wall formation, were investigated for their roles in storing drugs and controlling the release of drugs through the diffusion process. ${ }^{49}$ In vitro release tests indicated that SLS-formed devices were capable of retarding the release of model drugs into a simulated environment. Although non-degradable reservoir-type DDDs can provide zero-order release with the diffusion of drugs through the shell, they are hardly used in regenerative medicine. By using biodegradable polymers, however, DDDs made by SLS can be used for controlled drug/biomolecule release. PCL with methylene blue was sintered into a DDD. ${ }^{50}$ The drug release profile could be modified by the addition of a barrier layer to the DDD, which acted to reduce the initial burst.

Generally, drugs are loaded either by blending with polymer powders before SLS (Method A) or by adsorption on DDD after SLS (Method C), as illustrated in Figure 3. For example, a PCL-progesterone (PG, a steroid hormone) drug delivery system was fabricated by sintering PCL and PG powder mixtures. ${ }^{51}$ Alternatively, drugs or biomolecules can be encapsulated in microspheres and the microspheres subsequently used for SLS (Method B, Figure 3). As a demonstration, our group encapsulated bovine serum albumin (BSA) in Ca-P/PHBV nanocomposite microspheres. ${ }^{52}$ The BSAloaded $\mathrm{Ca}-\mathrm{P} / \mathrm{PHBV}$ microspheres were then processed into $3 \mathrm{D}$ scaffolds with good dimensional accuracy via SLS. The nanocomposite microspheres served as protective carriers for BSA during SLS. In the in vitro release study, an initial BSA burst release was observed from surface molecules, which was followed by a slow release from the encapsulated BSA..$^{52}$ The BSA encapsulation efficiency was found to be relatively low due to the incorporation method, materials used, and SLS process.
Conventional SLS can be used for incorporating drugs, which are not heat-sensitive, and SSLS offers a better choice for incorporating heat-sensitive drugs or biomolecules. Scaffolds composed of poly(D,L-lactic acid) (PDLLA) and a protein were made via SSLS..$^{53}$ The protein in the scaffolds sintered at various laser intensities retained substantial activity after SSLS.

In tissue engineering, scaffolds alone may not cause spontaneous healing and regeneration of functional tissue due to the lack of biochemical inducements such as growth factors, which promote cell proliferation and differentiation. Although biomolecules could be incorporated in 3D scaffolds by sintering mixed powders of biomolecules and polymer granules or by sintering biomolecule-loaded microspheres, SLS may cause severe damage to biomolecules due to the heat generated. One approach to avoid this problem is to bind biomolecules to scaffolds after their fabrication by SLS. In our study, in order to better control the growth factor release behavior, surface modification of sintered Ca-P/PHBV scaffolds was first conducted through physical entrapment of gelatin and subsequent immobilization of heparin. ${ }^{25}$ Recombinant human bone morphogenetic protein (rhBMP)-2 was then loaded on the surface modified scaffolds (Method D in Figure 3). The rhBMP-2 could be released in a controlled and sustained manner due to the specific affinity between heparin and rhBMP-2. This strategy not only provided a means to protect the loaded rhBMP-2 but also improved the rhBMP-2 release behavior.

In the subsequent in vitro study, the pluripotent mesenchymal stem cell line $\mathrm{C} 3 \mathrm{H} 10 \mathrm{~T} 1 / 2$ was seeded on bare $\mathrm{Ca}-\mathrm{P} / \mathrm{PHBV}$ scaffolds and surface modified Ca-P/PHBV scaffolds loaded with rhBMP-2. Proliferating cells were observed to cover all surfaces of surface modified and rhBMP-2 loaded scaffolds (Figure 4a). At a higher magnification, the formation of a Ca-P (or apatite) layer on some Ca-P-PHBV microspheres of the scaffold strut surface was also observed (Figure $4 b$ ), and there appeared to be interactions between cells and the Ca-P covered microspheres. In in vivo experiments, Ca-P/PHBV scaffolds with or without rhBMP-2 were implanted in a drilled hole in the ilium of New Zealand white rabbits. Six weeks after implantation, micro-CT data showed that there was very limited bone formation in Ca-P/PHBV scaffolds without rhBMP-2 loading (Figure 5a), with the defect (the drilled hole) being occupied by the scaffold. In contrast, new bone was formed adjacent to and inside the surface modified and rhBMP-2 loaded scaffold (Figure 5b). Histological examination showed that with surface modified and rhBMP-2 loaded scaffold, the center of the defect (the drilled hole) was filled with newly formed bone (Figure 6c), whereas there was no bone formation in the center of the defect treated with a scaffold without rhBMP-2, and new bone formation was stimulated only at the periphery of the defect (Figure 6a). Bone remodeling, or the replacement of old bone with new, which exhibited the light pink color in Figure 6d, was also 

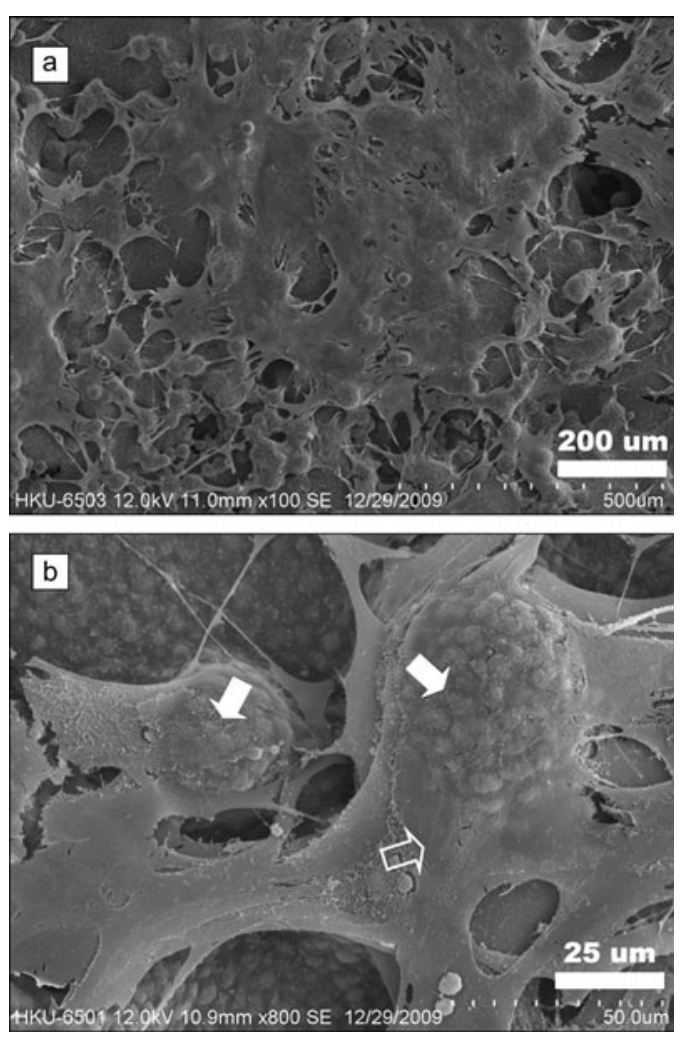

Figure 4. C3H10T1/2 cells cultured on a surface-modified and rhBMP-2 loaded Ca-P/PHBV nanocomposite scaffold for 21 days. (a) General view of cells on the scaffold. (b) A high magnification view. Solid white arrows indicate cell-covered Ca-P/PHBV microspheres; the hollow white arrow indicates a cell.

observed in the group treated with surface modified and rhBMP-2 loaded scaffolds. These investigations have demonstrated that the surface modification can provide binding sites for growth factors and control their sustained release in vitro and in vivo.

\section{Biomedical prototypes and devices}

When it was first borrowed from the manufacturing industry for biomedical applications, SLS was used to produce accurate models of elements such as the skull, mandible, and neuronetwork for simulation and surgical planning, particularly in the craniofacial and maxillofacial fields and for neurosurgery. A few engineering materials in powdered form, such as polycarbonate, nylon (standard nylon, fine nylon, fine nylon medical grade, and nylon composite) and acrylic, can be used to create these models. Using high-resolution, multiplanar imaging techniques such as MRI and CT, 3D medical image data can be obtained and used to reconstruct 3D models, which are utilized for fabrication via SLS. Physical models of the brain and skull were thus made using SLS for training in procedures of deep microvascular anastomosis. ${ }^{54}$ Using the fabricated model, neurosurgeons could practice with both realistic depth and width when manipulating operative instruments. The accuracy of biomedical prototypes is very important for surgical planning.
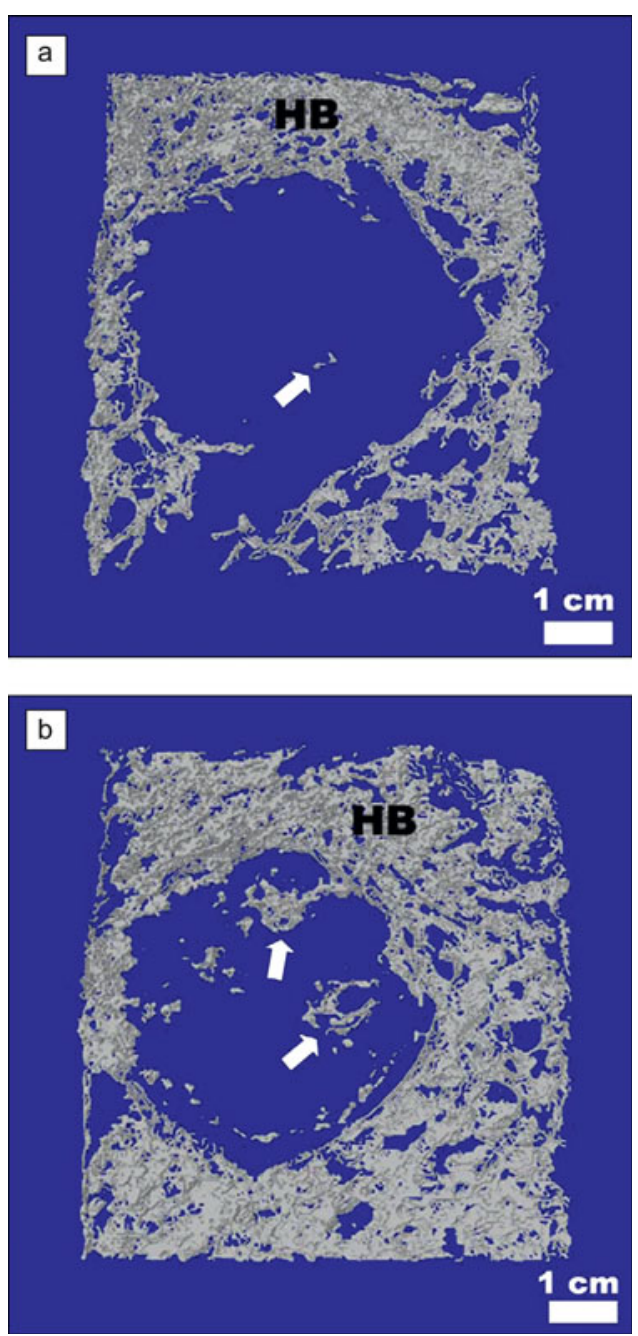

Figure 5. Micro-CT reconstructed images of scaffolds implanted in the ilium of rabbits for six weeks. (a) Ca-P/PHBV scaffold. (b) Surface-modified and rhBMP-2-loaded Ca-P/PHBV scaffold. HB, host bone. White arrows are pointing at newly formed bone within the scaffold in a drilled hole (defect).

The shape, dimensions, and anatomic details of prototypes may be affected by errors at any phase of the SLS process. Silva et al. analyzed the capability of SLS to reproduce craniomaxillary anatomy and compared the accuracy of an anatomical model made by SLS with that of a model fabricated by 3 DP. ${ }^{55}$ The SLS prototypes showed greater dimensional precision and reproduced craniomaxillary anatomy more accurately than the 3DP models. SLS prototypes also had a greater dimensional accuracy in reproducing mandibular anatomy than models produced by 3DP using a PolyJet machine. ${ }^{56}$

One distinctive advantage of RP techniques for biomedical engineering is the fabrication of customized medical devices, catering to the needs of individual patients. Using SLS, Wu et al. fabricated a wax pattern of a partial nasal prosthesis. ${ }^{57}$ Montgomery et al. designed an actively actuated prosthetic socket (the portion of the prosthesis that attaches to the residual 

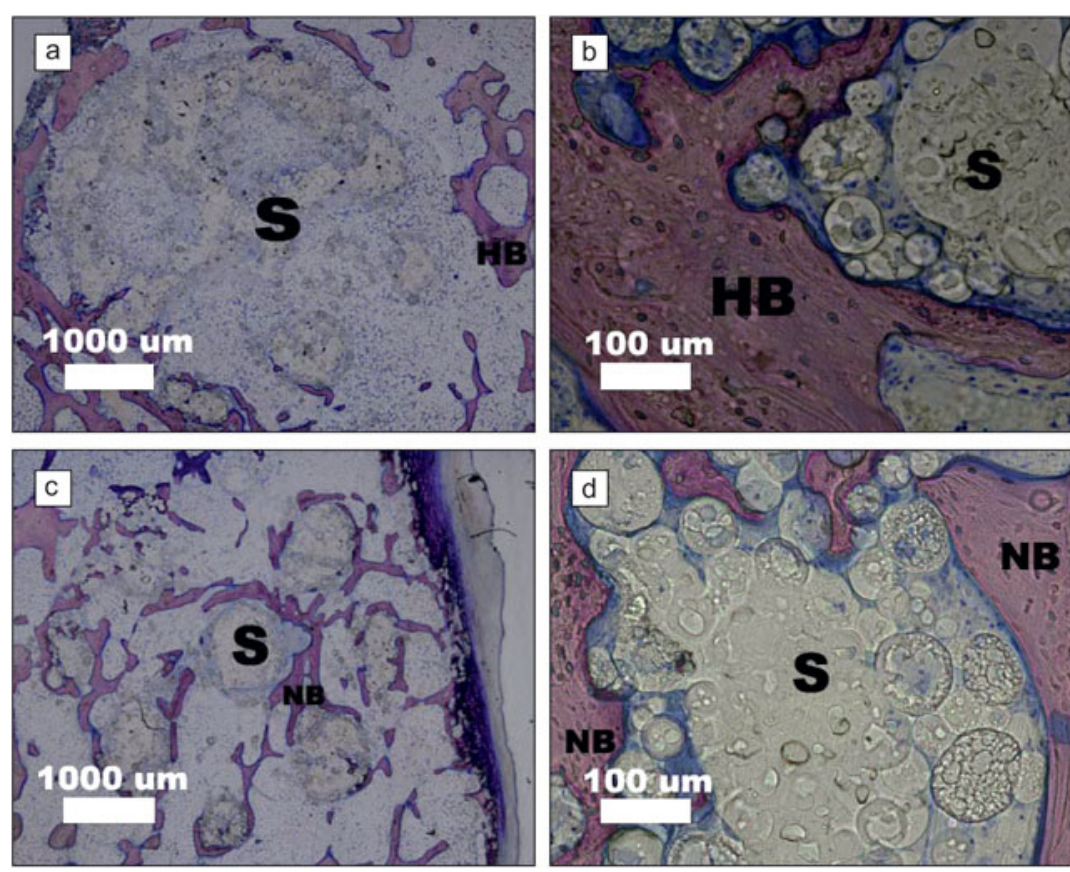

Figure 6. Histological images of tissues after scaffold implantation in the ilium of rabbits for six weeks: (a, b) Ca-P/PHBV nanocomposite scaffold; (c, d) surface-modified and rhBMP-2-loaded Ca-P/PHBV scaffold. S, scaffold; HB, host bone; NB, new bone. (a, c) General view; (b, d) close view.

Drug/biomolecule delivery systems can also be fabricated either by sintering drug and polymer blends or drug/biomolecule-containing microspheres or by adsorption of drug or noncovalent binding of biomolecules to SLS-formed delivery vehicles.

For tissue engineering, the control over mechanical properties and degradation behavior of sintered scaffolds is important. These properties are affected by the biodegradable materials used, scaffold design, and optimization of SLS parameters. One disadvantage of the SLS technique for scaffold fabrication is that hydrogels cannot be used, and it is also impossible to encapsulate cells in scaffolds. Using appropriate cell seeding techniques and advanced bioreactors should ensure that cells are homogeneously distributed on 3D scaffolds and also in pores inside the scaffolds.

The incorporation and release of chemotactic cues (e.g., growth factors) can facilitate the cell homing process. Our surface modified nanocomposite scaffolds with immobilized heparin can be loaded with two different growth factors, such as bone morphogenetic proteins, vascular endothelial growth factors, and fibroblast growth factors. The growth factors are

limb of an amputee and serves a variety of functions) and using nylon, manufactured the adaptive socket according to the design. ${ }^{58}$ Test results indicated great potential of the socket that could provide greater comfort and fit to avoid movement problems and rehabilitation difficulties. Another example of SLS applications is orthotic device fabrication. Using SLS, Faustini et al. manufactured ankle-foot orthoses (AFOs), which are assistive or therapeutic devices to improve the gait performance of people with impaired lower limb function. ${ }^{59}$ SLS allowed the fabrication of AFOs directly from digital shape information of a patient's limb and enabled the optimization of characteristics, such as weight, stiffness and shape, of AFOs. Customized foot orthoses, which lessen the symptoms of rheumatoid arthritis and improve function and mobility, have also been fabricated using SLS. ${ }^{60}$

\section{Summary and outlook}

SLS is a versatile RP technique that has exhibited great potential for biomedical applications. With careful modifications, commercial SLS machines can use small amounts of powdered materials to produce designed objects for specific biomedical applications. It has been shown by different research groups that optimization of SLS parameters is of great importance for fabricating designed objects with good quality and high accuracy. Using data obtained from medical imaging techniques such as MRI and CT, anatomically shaped tissue engineering scaffolds with specifically designed architectures, customized medical devices, and surgical models can be produced via SLS. expected to be released independently in a controlled and sustained manner independently and to stimulate the tissue regeneration in an orchestrated way. The lack of vascularization within the scaffold is still a major issue for 3D scaffolds targeted at specific tissue regeneration. The dual growth factor delivery approach can overcome this by stimulating angiogenesis, nourishing the newly formed tissue, and improving in vivo integration with host tissue. Apart from loading two different growth factors on scaffolds, the dual release approach can be employed to encapsulate drugs in scaffolds in combination with loading growth factors on the surface. Some small-molecule drugs such as antibiotics and anti-inflammation drugs can be incorporated in SLS-formed scaffolds, as they may be able to withstand the SLS process. A local delivery system providing a sustained release of sufficient doses of antibiotics after surgery has clinical benefits in preventing infections during the treatment of bone defects or bone fractures.

SLS is capable of fabricating objects with gradient structures. ${ }^{61-63}$ Sintered functionally graded scaffolds could have potential applications in interface tissue engineering (e.g., osteochondral tissue engineering). With gradual changes in composition and structure, functionally graded scaffolds can mimic bone and cartilage properties and form the osteochondral interface. Currently, SLS can create multilayer functionally graded objects in which the material gradient is oriented along the build direction. Advanced SLS machines should be developed to automatically load different materials or materials of different compositions according to scaffold designs. With 
further development of SLS technology, the fabrication and use of complex scaffolds with gradient composition and/ or structure in three directions will greatly advance tissue engineering.

\section{Acknowledgments}

B. Duan thanks the University of Hong Kong (HKU) for the University Scholarship Award, which enabled him to study for his $\mathrm{PhD}$ at HKU. Our SLS research was supported by HKU through a research grant and by Hong Kong Research Grants Council through GRF grants (HKU 7182/05E and 7181/09E). Assistance provided by staff and students in the Department of Mechanical Engineering and Department of Orthopaedics and Traumatology, HKU, is acknowledged.

\section{References}

1. K.F. Leong, C.M. Cheah, C.K. Chua, Biomaterials 24, 2363 (2003)

2. S.F. Yang, K.F. Leong, Z.H. Du, C.K. Chua, Tissue Eng. 8, 1 (2002).

3. S.M. Peltola, F.P.W. Melchels, D.W. Grijpma, M. Kellomaki, Ann. Med. 40, $268(2008)$

4. S.J. Hollister, Adv. Mater. 21, 3330 (2009).

5. M.A. Lopez-Heredia, J. Sohier, C. Gaillard, S. Quillard, M. Dorget, P. Layrolle, Biomaterials 29, 2608 (2008).

6. W. Xu, X.H. Wang, Y.N. Yan, R.J. Zhang, J. Bioact. Compat. Polym. 23, 103 (2008).

7. T.B.F. Woodfield, M. Guggenheim, B. von Rechenberg, J. Riesle, C.A. van Blitterswijk, V. Wedler, Cell Proliferat. 42, 485 (2009)

8. W.Y. Zhou, S.H. Lee, M. Wang, W.L. Cheung, W.Y. Ip, J. Mater. Sci-Mater. Med. 19, 2535 (2008).

9. F.E. Wiria, N. Sudarmadji, K.F. Leong, C.K. Chua, E.W. Chng, C.C. Chan, Rapid Prototyping J. 16, 90 (2010).

10. E.N. Antonov, V.N. Bagratashvili, S.M. Howdle, A.N. Konovalov, V.K. Popov, V.Y. Panchenko, Laser Phys. 16, 774 (2006).

11. T.B. Bukharova, E.N. Antonov, V.K. Popov, T.K. Fatkhudinov, A.V. Popova, A.V. Volkov, S.A. Bochkova, V.N. Bagratashvili, D. Gol'dshtein, Bull. Exp. Biol. Med. 149, 148 (2010).

12. D.T. Pham, S. Dimov, F. Lacan, Proc. Inst. Mech. Eng. Part B-J. Eng. Manuf. 213, 435 (1999).

13. M.M. Savalani, L. Hao, R.A. Harris, Proc. Inst. Mech. Eng. Part B-J. Eng. Manuf. 220,171 (2006)

14. N.K. Tolochko, V.V. Savich, T. Laoui, L. Froyen, G. Onofrio, E. Signorelli, V.I. Titov, Proc. Inst. Mech. Eng. Pt. L-J. Mater. Des. Appl. 216, 267 (2002).

15. I.V. Shishkovsky, L.T. Volova, M.V. Kuznetsov, Y.G. Morozov, I.P. Parkin, J. Mater. Chem. 18, 1309 (2008).

16. R. Comesana, F. Lusquinos, J. del Val, T. Malot, M. Lopez-Alvarez, A. Riveiro, F. Quintero, M. Boutinguiza, P. Aubry, A. De Carlos, J. Pou, J. Eur. Ceram. Soc. 31, 29 (2011).

17. C. Shuai, C. Gao, Y. Nie, H. Hu, Y. Zhou, S. Peng, Nanotechnology 22, 285703 (2011).

18. H. Chung, S.P. Das, Mater. Sci. Eng., A 437, 226 (2006).

19. G. Salmoria, P. Klauss, R.A. Paggi, L.A. Kanis, A. Lago, Polym. Testing 28, 648 (2009)

20. L. Hao, M.M. Savalani, Y. Zhang, K.E. Tanner, R.A. Harris, Proc. Inst. Mech. Eng. Part H-J. Eng. Med. 220, 521 (2006).

21. F.E. Wiria, K.F. Leong, C.K. Chua, Y. Liu, Acta Biomater. 3, 1 (2007).

22. B. Partee, S.J. Hollister, S. Das, J. Manuf. Sci. Eng. 128, 531 (2006)

23. S. Eshraghi, S. Das. Acta Biomater. 6, 2467 (2010).

24. B. Duan, M. Wang, W.L. Cheung, Biofabrication 3, 015001 (2011)

25. B. Duan, M. Wang, J. R. Soc. Interface 7, S615 (2010).

26. R. Langer, J.P. Vacanti, Science 260, 920 (1993).

27. D.W. Hutmacher, S. Cool, J. Cell. Mol. Med. 11, 654 (2007).
28. W.Y. Yeong, C.K. Chua, K.F. Leong, M. Chandrasekaran, Trends Biotechnol. 22, 643 (2004).

29. J.T. Rimell, P.M. Marquis, J. Biomed. Mater. Res. 53, 414 (2000).

30. M. Schmidt, D. Pohle, T. Rechtenwald, CIRPAnn. Manuf. Technol. 56, 205 (2007) 31. L. Hao, M.M. Savalani, Y. Zhang, K.E. Tanner, R.A. Harris, Proc. Inst. Mech. Eng. Pt. L-J. Mater. Des. Appl. 220, 125 (2006).

32. K.H. Tan, C.K. Chua, K.F. Leong, C.M. Cheah, P. Cheang, M.S. Abu Bakar, S.W. Cha, Biomaterials 24, 3115 (2003).

33. Y. Zhang, L. Hao, M.M. Savalani, R.A. Harris, L. Di Silvio, K.E. Tanner, J. Biomed. Mater. Res. Part A 91A, 1018 (2009).

34. K.H. Tan, C.K. Chua, K.F. Leong, M.W. Naing, C.M. Cheah, Proc. Inst. Mech. Eng. Part H-J. Eng. Med. 219, 183 (2005).

35. M.A. Woodruff, D.W. Hutmacher, Prog. Polym. Sci. 35, 1217 (2010).

36. J.M. Williams, A. Adewunmi, R.M. Schek, C.L. Flanagan, P.H. Krebsbach, S.E. Feinberg, S.J. Hollister, S. Das, Biomaterials 26, 4817 (2005).

37. G. Ciardelli, V. Chiono, G. Vozzi, M. Pracella, A. Ahluwalia, N. Barbani, C. Cristallini, P. Giusti, Biomacromolecules 6, 1961 (2005).

38. K.H. Tan, C.K. Chua, K.F. Leong, C.M. Cheah, W.S. Gui, W.S. Tan, F.E. Wiria, Bio-Med. Mater. Eng. 5, 113 (2005).

39. R.L. Simpson, F.E. Wiria, A.A. Amis, C.K. Chua, K.F. Leong, U.N. Hansen, M. Chandraselkaran, M.W. Lee, J. Biomed. Mater. Res. Part B 84B, 17 (2008).

40. F.E. Wiria, C.K. Chua, K.F. Leong, Z.Y. Quah, M. Chandrasekaran, W. Lee, J. Mater. Sci.-Mater. Med. 19, 989 (2008).

41. J.J. Li, Y. Dou, J. Yang, Y.J. Yin, H. Zhang, F. Yao, H.B. Wang, K.D. Yao, Mater. Sci. Eng., C 29, 1207 (2009).

42. S.J. Heo, S.E. Kim, J. Wei, Y.T. Hyun, H.S. Yun, D.H. Kim, J.W. Shin, J. Biomed. Mater. Res. Part A 89A, 108 (2009).

43. B. Duan, M. Wang, W.Y. Zhou, W.L. Cheung, Z.Y. Li, W.W. Lu, Acta Biomater. 6, $4495(2010)$

44. B. Duan, M. Wang, Z.Y. Li, W.C. Chan, W.W. Lu, Front. Mater. Sci. 5, 57 (2011).

45. J. Liu, B. Zhang, C.Z. Yan, Y.S. Shi, Rapid Prototyping J. 16, 138 (2010).

46. W.Y. Yeong, N. Sudarmadji, H.Y. Yu, C.K. Chua, K.F. Leong, S.S. Venkatraman, Y.C.F. Boey, L.P. Tan, Acta Biomater. 6, 2028 (2010).

47. H. Huang, S. Oizumi, N. Kojima, T. Niino, Y. Sakai, Biomaterials 28 $3815(2007)$

48. K.F. Leong, K.K.S. Phua, C.K. Chua, Z.H. Du, K.O.M. Teo, Proc. Inst. Mech. Eng. Part H-J. Eng. Med. 215, 191 (2001).

49. C.M. Cheah, K.F. Leong, C.K. Chua, K.H. Low, H.S. Quek, Proc. Inst. Mech. Eng. Part H-J. Eng. Med. 216, 369 (2002).

50. K.F. Leong, F.E. Wiria, C.K. Chua, S.H. Li, Bio-Med. Mater. Eng. 17, 147 (2007).

51. C.V. Salmoria, P. Klauss, R.A. Paggi, M. Souza, L.A. Kanis, K.M. Zepon, Innovative Developments in Design and Manufacturing: Advanced Research in Virtual and Rapid Prototyping (CRC Press, 2010), p. 229.

52. B. Duan, M. Wang, Polym. Degrad. Stabil. 95, 1655 (2010).

53. E.N. Antonov, V.N. Bagratashvili, M.J. Whitaker, J.J.A. Barry, K.M. Shakesheff, A.N. Konovalov, V.K. Popov, S.M. Howdle, Adv. Mater. 17, 327 (2005).

54. M. Wanibuchi, M. Ohtaki, T. Fukushima, A.H. Friedman, K. Houkin, Acta Neurochir. 152, 1055 (2010).

55. D.N. Silva, M.G. De Oliveira, E. Meurer, M.I. Meurer, J.V.L. Da Silva, A. Santa-Barbara, J. Cranio-Maxillofac. Surg. 36, 443 (2008).

56. D. Ibrahim, T.L. Broilo, C. Heitz, M.G. de Oliveira, H.W. de Oliveira, S.M.W. Nobre, J.H.G. Dos Santos, D.N. Silva, J. Cranio-Maxillofac. Surg 37, 167 (2009).

57. G.F. Wu, B. Zhou, Y.P. Bi, Y.M. Zhao, J. Prosthet. Dent. 100, 56 (2008).

58. J.T. Montgomery, M.R. Vaughan, R.H. Crawford, Rapid Prototyping J. 16, 194 (2010).

59. M.C. Faustini, R.R. Neptune, R.H. Crawford, S.J. Stanhope, IEEE Trans. Biomed. Eng. 55, 784 (2008).

60. J.H.P. Pallari, K.W. Dalgarno, J. Woodburn, IEEE Trans. Biomed. Eng. $\mathbf{5 7}$ 1750 (2010).

61. H. Chung, S. Das, Mater. Sci. Eng., A 487, 251 (2008).

62. J.L. Leite, G.V. Salmoria, R.A. Paggi, C.H. Ahrens, A.S. Pouzada, Int J. Mater. Prod. Technol. 39, 205 (2010).

63. N. Sudarmadji, J.Y. Tan, K.F. Leong, C.K. Chua, Y.T. Loh, Acta Biomater. 7 , 530 (2011).
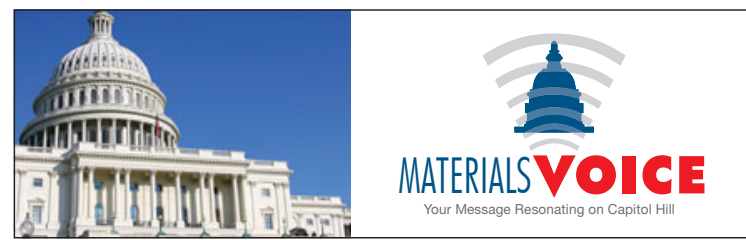

A Web-based tool to ensure that your voice is heard on Capitol Hill www.mrs.org/materialsvoice 